\title{
Realidade Aumentada em Livros Infantis: uma revisão sistemática de pesquisas e aplicações
}

\author{
Roberta Gerling Moro ${ }^{1}$ \\ ${ }^{1}$ Núcleo de Estudos e Pesquisas sobre Cultura Escrita Digital - Universidade Federal de \\ Minas Gerais (UFMG) - Belo Horizonte, MG - Brazil \\ robgmoroegmail. com
}

\begin{abstract}
This article aims to present a systematic review of research and applications of augmented reality in children's books. A bibliographical survey was carried out in the main international databases in the period from 2012 to 2017. For this purpose, 21 researches were selected for the analyzes. The results confirm the playful and immersive character provided by AR technology, collaborating in the learning performance of children during reading practices.
\end{abstract}

Keywords: Children's books; Systematic Literature Review; Augmented Reality.

Resumo. Este artigo tem como objetivo apresentar uma revisão sistemática de pesquisas e aplicações da realidade aumentada em livros infantis. Para isso, foi realizado um levantamento bibliográfico nas principais bases internacionais no periodo de 2012 a 2017. Para a pesquisa, foram selecionados 21 estudos que abordam o tema. Os resultados das análises confirmam o caráter lúdico e imersivo proporcionados pela tecnologia $R A$, colaborando no desenvolvimento da aprendizagem de crianças durante as práticas de leitura.

Palavras-chave: Livros Infantis; Revisão Sistemática de Literatura; Realidade Aumentada

\section{Introdução}

O crescente investimento da tecnologia na área educacional, principalmente a partir da popularização dos dispositivos móveis, fez com que o mercado editorial voltasse sua atenção para a produção de obras digitais infantis. Devido à pouca atratividade desempenhada pelos e-books, o mercado editorial também vem investindo em um formato com um maior nível de interatividade e complexidade, é o caso dos app books, também conhecidos como book apps, aplicativos literários, livros-aplicativos ou livros digitais interativos, como são comumente denominados. Tais versões podem ser adquiridas exclusivamente através das app stores da Google Play ou da Apple. Os book apps podem ser descritos como versões aprimoradas dos livros infantis impressos que podem trazer, em sua estrutura interna, conteúdos adicionais, características e opções de navegação não relacionadas diretamente com o conteúdo do formato físico. Animações, 
VII Congresso Brasileiro de Informática na Educação (CBIE 2018)

Anais do XXIX Simpósio Brasileiro de Informática na Educação (SBIE 2018)

efeitos sonoros, narração automática e hiperlinks que direcionam o leitor a conteúdos externos à narrativa, são apenas alguns elementos que podem ser encontrados nestas versões digitais [Serafini et al., 2015].

Como é possível perceber, os livros infantis têm alcançado níveis significativos na exploração dos recursos computacionais para a promoção da leitura, como a implementação de vídeos, jogos, interatividade, realidade virtual e aumentada. Entretanto, ainda há poucos estudos que abordam a realidade aumentada como recurso integrado aos livros infantis, aspecto que será abordado neste trabalho.

A maioria das experiências voltadas à realidade aumentada tem focado apenas nos aspectos visuais e na exploração de outros recursos, como áudio ou interfaces de transição. Um dos principais objetivos ao se inserir tais recursos é criar uma experiência mais imersiva para o usuário. Contudo, um dos maiores potenciais da realidade aumentada é a integração da interatividade. No caso dos livros infantis, a interação inicia na medida em que o usuário manipula o livro físico (interface material) para então, explorar o conteúdo virtual de diferentes posições e páginas [Clark \& Dünser, 2012]. Pode ser citado como exemplo, a obra The Mouse and the Meadow, do autor e ilustrador Chad Wallace (2014). A partir do livro físico, foi criado o aplicativo $3 D$ popup, onde a criança pode visualizar os personagens e cenários em animações tridimensionais. Além disso, o leitor pode acompanhar a leitura através da narração automática e interagir com os elementos da história.

Altinpulluk e Kesim (2016) realizaram uma revisão sistemática a respeito dos livros com realidade aumentada, coletando pesquisas acadêmicas a fim de classificar e avaliar os tipos de livros e tecnologias utilizadas. O presente trabalho, por outro lado, propõe uma revisão sistemática com o intuito de verificar os estudos que estão sendo realizados no contexto da realidade aumentada aplicada nos livros infantis, os quais englobam a produção de obras, recursos empregados, análise de pesquisas empíricas realizadas com crianças, reações e tipos de motivação por parte dos leitores infantis frente a essas obras. Para isso, o presente trabalho está estruturado em quatro seções principais, além desta breve introdução. Na segunda seção, é apresentada a metodologia de revisão sistemática empregada; posteriormente, na terceira seção, são analisados os dados obtidos a partir desta revisão; e, por fim, são apontadas algumas conclusões a que foi possível chegar com este trabalho.

\section{Metodologia}

A metodologia utilizada para a coleta e análise de pesquisas com o objetivo de identificar os conceitos e desafios, bem como a relevância do estudo de livros infantis com realidade aumentada, busca respaldo na revisão sistemática de literatura (systematic literature review) (SLR). A SLR é utilizada para identificar, avaliar e interpretar os dados disponibilizados dentro de um período e campo de pesquisa específicos [Ramírez; Peñalvo, 2018]. Embora existam inúmeras metodologias para a revisão sistemática, neste trabalho, optou-se por seguir as três etapas indicadas por Kitchenham \& Charters (2007) e adaptadas de Neiva e Silva (2016), a saber (1) planejamento; (2) execução; (3) extração, conforme descritas a seguir. 
VII Congresso Brasileiro de Informática na Educação (CBIE 2018)

Anais do XXIX Simpósio Brasileiro de Informática na Educação (SBIE 2018)

2.1. Planejamento: na primeira etapa, foi definida a delimitação temporal, nesse caso, optou-se pelo período de 2012 a 2017. Dentro desse período, buscou-se responder, em um primeiro momento, a uma questão mais ampla (Quais estudos têm sido realizados sobre a realidade aumentada integrada ao livro infantil?) que fomentou a formulação de questões mais específicas, listadas abaixo, as quais possibilitaram um maior refinamento nas buscas:

(Q1): Em quais países se concentram as pesquisas sobre a literatura infantil com realidade aumentada?

(Q2): Quais são as principais classificações dos livros infantis com realidade aumentada? O que tem sido produzido especificamente para este público?

(Q3): Qual é o foco das pesquisas integrando a RA nos livros infantis?

(Q4): Como as crianças leem os livros literários aumentados? A realidade aumentada pode ser um fator de motivação durante a leitura?

Para a busca, foram selecionadas as principais bases internacionais, de forma a investigar a temática de uma forma mais ampla, em âmbito nacional e internacional, como Google Acadêmico, IEE, Scopus, Springer, Science Direct, Ebsco e ACM. Os argumentos de busca utilizados são variantes e sinônimos dos termos augmented AND reality AND books AND for AND children (augmented reality AND children's literature; augmented reality in literature for children; augmented reality in picture books; augmented reality AND children's books; children interacting with augmented reality storybooks). Nesta busca inicial, foram recuperadas 2111 pesquisas, entre teses, dissertações e artigos publicados em periódicos e conferências.

2.2. Execução: Alguns refinamentos foram necessários nesta etapa, já que muitos dos estudos coletados estavam relacionados apenas à realidade aumentada nas áreas da educação, ciências, saúde e psicologia, o que afastava, de certa forma, do foco da pesquisa. Nesse sentido, foram incluídos trabalhos que: a) foram realizados entre $2012 \mathrm{e}$ 2017; b) apresentam análise qualitativa; c) abordam livros infantis com RA; d) apresentam perspectivas teóricas sobre a RA aplicada nos livros infantis; e) apresentam resultados a partir de estudos empíricos realizados com crianças; f) analisam recursos, livros infantis e livros de imagem (augmented reality picture books); g) Apresentam contribuições relevantes para o campo analisado. Por outro lado, foram excluídas as pesquisas que: a) foram realizadas antes de 2012; b) não estão em inglês, espanhol ou português; c) apresentam análise de materiais educacionais e livros didáticos; d) priorizam a RA e outras áreas não relacionadas com o estudo de obras infantis; e) Não estão disponibilizados para download.

$\mathrm{Na}$ etapa de execução, as pesquisas foram baixadas em arquivo BibText. e armazenadas em um software de revisão sistemática, o StArt (versão beta 3). A partir da leitura dos títulos, palavras-chave e resumos, das 2111 pesquisas, foram recuperadas apenas 35. Partindo dos critérios citados anteriormente, uma leitura mais aprofundada foi necessária para verificar a relevância dos estudos coletados, sendo selecionadas para a análise 21 pesquisas.

2.3. Extração: A terceira e última etapa foi dedicada à apresentação de uma síntese a partir dos dados coletados. Na tabela abaixo, estão indicados o período, a classificação 
VII Congresso Brasileiro de Informática na Educação (CBIE 2018)

Anais do XXIX Simpósio Brasileiro de Informática na Educação (SBIE 2018)

dos trabalhos selecionados (artigo de periódico/conferência, teses ou dissertações) e a quantidade de publicações. Ressalta-se que os trabalhos duplicados, bem como aqueles que apresentavam pouca contribuição na análise de livros infantis com RA e que não permitiam uma leitura completa, ou seja, não estavam disponíveis online e tampouco podiam ser baixados, foram excluídos da seleção.

Tabela 1. Quantidade de artigos selecionados por ano e classificação

\begin{tabular}{|c|c|c|c|c|}
\hline Ano & $\begin{array}{c}\text { Artigos de } \\
\text { periódicos }\end{array}$ & $\begin{array}{c}\text { Artigos de } \\
\text { eventos/conferências }\end{array}$ & Dissertações & Teses \\
\hline 2012 & & 2 & 1 & \\
\hline 2013 & & 2 & 1 & \\
\hline 2014 & 5 & 1 & & \\
\hline 2015 & 2 & & 1 & \\
\hline 2016 & 2 & & & 1 \\
\hline 2017 & 2 & 1 & $\mathbf{3}$ & $\mathbf{1}$ \\
\hline Total & $\mathbf{1 1}$ & $\mathbf{6}$ & & \\
\hline
\end{tabular}

\section{Análise dos resultados}

Nas subseções a seguir, apresentam-se análises mais específicas, as quais estão organizadas por categorias, a saber, (1) países, nessa categoria pretende-se apresentar os países com maior concentração de pesquisas; (2) produção de livros infantis com RA, aqui, verifica-se quais tipos de obras têm sido produzidas para o público infantil; (3) foco das pesquisas, apresentam-se os principais focos das pesquisas selecionadas; (4) práticas de leitura e motivação, esta categoria pretende responder, por fim, ao seguinte questionamento "Como as crianças leem os livros literários infantis com RA?", tentando relacionar aos principais fatores de motivação expressos pelas crianças durante as práticas de leitura.

\subsection{Países}

Ao realizar a contabilização e menções das afiliações dos autores de cada pesquisa, a tabela 2 permite verificar a localidade que se concentram as pesquisas selecionadas. Percebe-se uma maior concentração nos países pertencentes à Ásia (10) e Europa (6), sendo o Brasil e o Taiwan os países que mais renderam pesquisas sobre a RA aplicada na literatura infantil. Dessa forma, é possível concluir que ainda há pouco investimento de pesquisas sobre RA e livros infantis tanto no contexto nacional, quanto internacional, conforme indicado na tabela. 
VII Congresso Brasileiro de Informática na Educação (CBIE 2018)

Anais do XXIX Simpósio Brasileiro de Informática na Educação (SBIE 2018)

Tabela 2. Países de afiliação dos autores das pesquisas

\begin{tabular}{|c|c|}
\hline Países & Número de pesquisas \\
\hline Brasil & 3 \\
\hline Estados Unidos & 1 \\
\hline Noruega & 2 \\
\hline Portugal & 1 \\
\hline Inglaterra & 1 \\
\hline Espanha & 1 \\
\hline Suíça & 1 \\
\hline Nova Zelândia & 1 \\
\hline Malásia & 2 \\
\hline Índia & 1 \\
\hline China & 2 \\
\hline Coréia & 1 \\
\hline Taiwan & 3 \\
\hline Turquia & 1 \\
\hline & \\
\hline
\end{tabular}

\subsection{Produção de livros infantis com RA}

Do número total das pesquisas, 16 trazem como material de análise a produção de livros infantis aumentados. Dentre as pesquisas analisadas, foi possível identificar quatro classificações principais, as quais são: (1) protótipos; (2) adaptações; (3) criações; (4) livros de colorir.

É interessante verificar que apenas duas pesquisas apresentam protótipos de livros infantis aumentados [Eiksund, 2012; Clark \& Dünser, 2012], sendo a maioria das pesquisas voltadas para a adaptação de livros físicos (poesias, clássicos, fábulas, livros de imagem), implementados, posteriormente, à tecnologia RA. Nessa categoria, é possível encontrar a adaptação de histórias de folclore, como "The Thirsty Crow" e "Sang Kancil and the Crocodiles" para o desenvolvimento de uma interface gráfica interativa para a aprendizagem de números em RA [Rambli et al., 2012; Tomi \& Rambli, 2013], e a obra "L.I.Neu e o enigma das letras", publicado pela editora UFRN, para o aprendizado do alfabeto [Naschold et al., 2015]. Outras pesquisas realizaram adaptações a partir de obras literárias infantis, como é o caso dos livros "O que Lola quer, Lola tem", "Lino, o Limpossauro", "Dinosaurs Alive" e "Fairyland Magic", das editoras Scribblers em parceria com The Salariya Book Company e Carlton Books [Ribeiro, 2013]. Há outros estudos que também se valeram de adaptações de clássicos, como a fábula "Sun and Wind", de Esopo [Gil et al., 2014] e livros de imagem sobre artistas e obras de arte, como "The Adventures of Yuyu: Yuyu Yang Artistic Journey" [Cheng et al., 2014; Cheng, 2017]. 
VII Congresso Brasileiro de Informática na Educação (CBIE 2018)

Anais do XXIX Simpósio Brasileiro de Informática na Educação (SBIE 2018)

Apenas quatro pesquisas apresentam criações artístico-literárias para a implementação de RA voltada para a leitura. Duas delas trazem criações colaborativas, uma realizada a partir do desenvolvimento de livros físicos e implementação da tecnologia RA com softwares específicos, como o ARtool-Book e Audacity, este último para a inserção de recursos sonoros [Kelling, 2016]; e a outra se refere ao desenvolvimento de livros e textos colaborativos produzidos a partir da integração de crianças e adultos e aplicação, posteriormente, da RA [Alhumaidan, 2017]. Nessa categoria, também foram encontradas pesquisas que apresentam a criação de livros de imagens, produzidos pelos próprios autores dos estudos [Yalmaz et. al, 2016; Zambonato \& Dick, 2016].

Em relação aos livros de colorir, somente três pesquisas abordam a temática focando-se especificamente no aspecto educacional dos livros e na geração de conteúdos tridimensionais a partir da pintura do usuário (criança) no livro físico [Clark \& Dünser, 2012]. Sobre o aspecto tridimensional, outras pesquisas investem na criação de uma aplicação dirigida à pintura de personagens por crianças em livros de colorir, em que o aplicativo se configura como um recurso para a criança verificar o resultado final de sua pintura [Magnenat et al., 2015; Zhao et al., 2017].

Por fim, verificou-se que apenas uma pesquisa apresenta uma obra literária infantil adaptada e implementada à tecnologia RA a partir de um aplicativo próprio da narrativa, como é o caso da obra "The Fantastic Flying Books of Mr. Morris Lessmore", em que a prática de leitura necessita da utilização simultânea de duas tecnologias distintas: o livro e o aplicativo com recursos RA. Aqui, a tecnologia RA é subordinada ao livro em seu formato físico, sendo as imagens bidimensionais e achatadas do livro transformadas em elementos tridimensionais, não alterando, contudo, o contexto original da história, apenas expandindo de um formato a outro [Schwebs, 2014].

\subsection{Foco das pesquisas}

Nos estudos selecionados, foram verificados dois focos recorrentes nas pesquisas, os quais são: (1) técnicas e ferramentas utilizadas e aplicadas para o engajamento dos usuários com os livros (elaboração de sistemas, técnicas, critérios para a seleção e aplicação de ferramentas, processos relacionados ao design de livros e implementação da tecnologia); (2) aspectos relacionados à interação das crianças com livros RA (compreensão leitora, fator de fomento e motivação para as atividades de leitura nas escolas, criação de textos colaborativos, leitura multimodal, concepções dos pais sobre o aprendizado das crianças com livros RA, implementação de RA voltada para a demanda de crianças em bibliotecas).

De um modo geral, os dois principais focos identificados parecem estar bem distribuídos entre as pesquisas selecionadas. Nota-se, no entanto, que apenas um artigo aborda questões mais conceituais sobre um livro RA em específico [Schwebs, 2014]. A maioria das pesquisas estão focadas prioritariamente nas técnicas, ferramentas e procedimentos adotados para a criação e aplicação de livros RA e em como as crianças reagiram à esta nova tecnologia durante a leitura.

A partir da análise das pesquisas, foi possível constatar a grande variedade existente de técnicas e ferramentas RA que podem ser aplicadas aos livros infantis. Uma 
VII Congresso Brasileiro de Informática na Educação (CBIE 2018)

Anais do XXIX Simpósio Brasileiro de Informática na Educação (SBIE 2018)

ferramenta mencionada com frequência nos estudos é a ARToolKit, comumente utilizada no desenvolvimento de sistemas RA [Yang, 2014]. São citadas também, como possibilidades de aplicação as técnicas e protótipos empregados no contexto da realidade aumentada para dispositivos móveis, como multimedia interactive book (miBook), Magical Playbook, mobile applications (aplicativos RA para dispositivos móveis), BuildAR Pro, Playspace, 3D-Puppetry system, sistemas baseados no rastreamento do ambiente físico (CSCIS system), Dialog book system e sistema para a produção de histórias interativas a partir da utilização de diferentes ferramentas e técnicas (mixed-reality interactive storytelling) [Aurelia et al., 2014].

Além desses sistemas, foram mencionados os recursos RA que podem ser aplicados na leitura de livros infantis e em situações de aprendizagem de uma forma geral, tais como: Aurasma (aplicação móvel multiplataforma); Augment (pode exportar formatos de outros programas, como o SketchUp; Quiver (aplicação baseada em realidade aumentada e virtual); Chromville (semelhante ao Quiver, sendo as criações aumentadas a partir da utilização da câmera do dispositivo); Zookazam (é possível adicionar diferentes espécies de animais para a criação de cenários de fábulas); Layar (aplicação móvel que possibilita o escaneamento de objetos, imagens e páginas de livros). Complementos para a obtenção de modelos tridimensionais também podem ser adquiridos através de plataformas da web (Warehouse 3D, TurboSquid, Archive 3D) e softwares de desenho gráfico e modelagem (SketchUp, Blender, entre outros) [Martínez \& López, 2017].

No que tange ao segundo foco das pesquisas, há uma grande preocupação por parte dos pesquisadores em verificar os tipos de reações e atitudes expressas no momento da leitura de livros RA. Dos dez trabalhos analisados, apenas um indica como resultado as principais concepções dos pais sobre o aprendizado das crianças com livros RA. A maioria dos trabalhos exploram as interações das crianças com os livros, com atenção especial à compreensão leitora, às motivações geradas pela tecnologia RA e se promove ou não o fomento à leitura. Um outro artigo relata sobre a utilização da RA como complemento para os sistemas tradicionais das bibliotecas, os quais são baseados em sistemas alfanuméricos e/ou em coleções únicas por tamanhos. Desse modo, a pesquisa contribui para o campo na medida em que indica o sistema RA como referência para a criança pesquisar por um livro de preferência nas bibliotecas, sem recorrer a um sistema, às vezes muito complexo, conforme o nível de alfabetização da criança. De acordo com a pesquisa, um sistema RA ideal permitiria às crianças se localizarem dentro de um mapa $3 \mathrm{D}$ da biblioteca/prédio. A presença de uma pesquisa por voz também facilitaria a busca dos exemplares, além de referências visuais (como a capa do livro), que poderiam ser utilizadas para direcionar os usuários ao conteúdo físico de sua preferência. Além disso, o sistema RA poderia fornecer uma opção para a exploração dos conteúdos não visíveis no ambiente físico da biblioteca. Em outros termos, o sistema RA poderia atuar como um "mentor-guia do leitor", o que poderia aumentar o número de crianças capacitadas para selecionar os livros desejados utilizando os seus próprios critérios como base da pesquisa [Meredith, 2014].

\subsection{Práticas de leitura e motivação}

Em se tratando dos questionamentos realizados na etapa do planejamento deste trabalho (como as crianças leem os livros literários aumentados? A realidade aumentada pode 
VII Congresso Brasileiro de Informática na Educação (CBIE 2018)

Anais do XXIX Simpósio Brasileiro de Informática na Educação (SBIE 2018)

ser um fator de motivação durante a leitura?), alguns aspectos sobre a interação das crianças com os livros RA que foram mencionados nas pesquisas, podem conduzir uma reflexão mais profunda sobre o modo como a tecnologia atuou nas práticas de leitura, tais como: as emoções positivas expressas pelas crianças durante a interação; o auxílio na compreensão dos textos; percepção do mundo físico; o modo com a RA afeta os pensamentos, sentimentos e comportamentos empáticos das crianças, entre outros. No que diz respeito à leitura compartilhada com os pais, as crianças pareciam se envolver mais e apresentar um desenvolvimento cognitivo maior em relação à compreensão da história. Por outro lado, quando as crianças manipulavam por conta própria, sua atenção voltava-se para a aparência dos elementos RA [Cheng \& Tsai, 2014].

Em outro artigo [Yalmaz et al., 2016], foi possível verificar que as crianças conceberam os livros RA como objetos "mágicos", ao imaginarem que os livros poderiam falar e que os personagens eram reais (vivos), provavelmente devido ao recurso tridimensional gerado pelas aplicações. Por este motivo, a maioria das pesquisas apontaram como fator de motivação as animações e personagens em 3D que geraram momentos lúdicos durante a leitura. Em contrapartida, as crianças tendem a focar sua atenção nesses recursos que promovem maior entretenimento e imersão, deixando de lado os elementos que propiciam o seu aprendizado.

\section{Considerações finais}

Neste trabalho, foram apresentadas algumas pesquisas e aplicações em torno do livro infantil com tecnologia RA. O desenvolvimento da pesquisa teve como escopo a verificação dos países de maior concentração, a produção de livros infantis, os focos das pesquisas e os modos como as crianças reagiram durante a leitura. A partir da análise de 21 pesquisas, conclui-se que o sistema RA pode facilitar a compreensão da história e aproximar a criança de aspectos mais complexos, como o aprendizado do alfabeto e dos números, bem como da leitura dos elementos artístico-literários dos livros de imagem.

Dessa forma, como foi apontado durante este trabalho, a mediação realizada por um adulto, assim como o planejamento de uma proposta que objetive o aprendizado e fomente a prática de leitura e artística são relevantes e devem ser considerados ao se inserir a tecnologia RA nos livros e imagens. As inúmeras técnicas e ferramentas mencionadas nas pesquisas, mostram o quanto é infinita e complexa a sua utilização e implementação, principalmente em se tratando de livros para crianças. Ao permitir a utilização simultânea do livro físico e de dispositivos digitais, a RA promove uma "reintegração" da materialidade do objeto, não excluindo, o que de certa forma ocorre com os livros digitais, o livro impresso. Sugere-se também, o aprimoramento das ilustrações do suporte físico - principalmente a inserção de elementos artísticos - os quais poderiam estimular a imersão e o prazer estético-literário nas crianças durante a leitura.

\section{Referências}

ALHUMAIDAN, Haifa. Co-Design of Augmented Reality Textbook for Children's Collaborative Learning Experience in Primary Schools. 2017. 184 f. Tese (Doctorate in Philosophy). Loughborough University. Inglaterra, 2017. 
VII Congresso Brasileiro de Informática na Educação (CBIE 2018)

Anais do XXIX Simpósio Brasileiro de Informática na Educação (SBIE 2018)

ALTINPULLUK, Hakan; KESIM, Mehmet. The classification of augmented reality books: a literature review. Proceedings of INTED 2016 Conference, Valencia, Espanha, p. 4110-4118, mar/2016.

AURELIA, P. Sagaya; RAJ, Durai; SALEH, Omer. A Survey on Mobile Augmented Reality Based Interactive, Collaborative and Location based Storytelling. Journal of Emerging Trends in Science and Technology, v. 1, n. 05, jul. 2014.

CHENG, Kung-Hung; TSAI, Chin-Chung. Children and parents' reading of an augmented reality picture book: Analyses of behavioral patterns and cognitive attainment. Computers \& Education, n. 72, p. 302-312, 2014.

CHENG, Kung-Hung. Exploring Parents' Conceptions of Augmented Reality Learning and Approaches to Learning by Augmented Reality With Their Children. Journal of Education Computing Research, p. 1-24, 2017.

CLARK, Adrian; DÜNSER, Andreas. An Interactive Augmented Reality Coloring Book. IEEE Symposium on 3D User Interfaces 2012, p. 7 - 10. USA, mar. 2012.

EIKSUND, Olaug. Children's Interaction with Augmented Reality Storybooks: a human-computer interaction study. 2012. 178 f. Dissertação (Department of Information Science and Media Studies) - University of Bergen. Bergen, 2012.

GIL, Kyungwon; RHIM, Jimin; HA, Taejin; DOH, Young Yim; WOOS, Woontack. AR PETITE THEATER: Augmented Reality Storybook for Supporting Children's Empathy Behavior. IEEE International Symposium on Mixed and Augmented Reality Media, Art, Social Science, Humanities and Design Proceedings, p. 13-20. Munich, 2014.

KELling, Véra Lúcia Vargas de Souza. Produção Textual e Multimodalidade: uma proposta com Realidade Aumentada. 2015. 103 f. Dissertação (Programa de PósGraduação em Tecnologias Educacionais em Rede) - Universidade Federal de Santa Maria. Santa Maria, 2015.

KITCHENHAM, Barbara., CHARTERS, Stuart. Guidelines for performing systematic literature reviews in software engineering (version 2.3). Technical report, Keele University and University of Durham, 2007.

MAGNENAT, Stéphane; NGO, Dat Tien Ngo; ZÜND, Fabio; RYFFEL, Mattia; NORIS, Gioacchino; ROTHLIN, Gerhard; MARRA, Alessia; NITTI, Maurizio; FUA, Pascal; GROSS, Markus; SUMMER, Robert W. Live Texturing of Augmented Reality Characters from Colored Drawings. IEEE Transactions on Visualization and Computer Graphics, v. 21, n. 11, p. 1201-1210. 2015.

MARTÍNEZ, Noelia Margarita Moreno; LÓPEZ, Juan Lucas Onieva. Herramientas y propuestas de innovación basadas en la tecnología de realidad aumentada aplicadas a la literatura infantil y juvenil. Tejuelo, n. 25, p. 217-244, 2017.

MEREDITH, Tamara R. Using Augmented Reality Tools to Enhance Children's Library Services. Technology, Knowledge and Learning, n. 20, n. 1, p. 71-77, abr. 2015.

NASCHOLD, Angela Chuvas; BALEN, Sheila; CAMPOS, André; SANTOS, Selan Rodrigues; SOLTOSKI, Michele; BRAZOROTO, Joseli; PEREIRA, Antonio. 
VII Congresso Brasileiro de Informática na Educação (CBIE 2018)

Anais do XXIX Simpósio Brasileiro de Informática na Educação (SBIE 2018)

Contando histórias com realidade aumentada: estratégia para promover a fluência da leitura infantil. Letras de Hoje, Porto Alegre, v. 59, n. 1, p. 138-146, jan.-mar. 2015.

NEIVA, Frâncila Weidt; SILVA, Rodrigo Luis de Souza. Systematic literature review in computer science: a practical guide. Technical report, Universidade Federal de Juiz de Fora, 2016.

RAMBLI, Dayang Rohaya; MATCHA, Wannisa; SULAIMAN, Suziah; NAYAN, Mohd Yunus. Design and Development of an Interactive Augmented Reality Edutainment Storybook for Preschool. International Conference on Future Computer Supported Education, p. 802 - 807, 2012.

RAMÍREZ, María-Soledad; PEÑALVO, Francisco-José García-. Co-creation and open innovation: Systematic literature review. Comunicar: Media Education Research Journal, n. 54, v. XXVI, p. 9-18, jan. 2018.

RIBEIRO, Sara Patrícia Patrão. Realidade Aumentada e suas implicações na promoção da leitura. 2013. 182 f. Dissertação (Mestrado em Ciências da Educação). Porto, 2013.

SCHWEBS, Ture. Affordances of an app: A reading of The Fantastic Flying Books of Mr. Morris Lessmore. Nordic Journal of ChildLit Aesthetics, v.5, 2014.

SERAFINI, Frank; KACHORSKY, Danielle; AGUILERA, Earl. Picturebooks 2.0: Transmedial Features Across Narrative Platforms. Journal of Children's Literature, v. 41, n. 2, p. 16-24, mar./jun. 2015.

TOMI, Azfar Bin; RAMBLI, Dayang Rohaya. An Interactive Mobile Augmented Reality Magical Playbook: Learning Number With The Thirsty Crow. International Conference on Virtual and Augmented Reality in Education, p. 123-130, 2013.

YANG, Wanggong. Research Of Augmented Reality For Children'S Books On The Basis Of Artoolkits. Applied Mechanics and Materials, v. 644-650, p. 3383-3386. Switzerland, 2014.

YILMAZ, Rabia M.; KUCUK, Sevda; GOKTAS, Yuksel. Are augmented reality picture books magic or real for preschool children aged five to six? British Journal of Educational Technology, p. 1-18, 2016.

ZAMBONATO, Augusto. DICK, Maurício Elias. A Realidade Aumentada como possibilidade de expansão à narrativa do livro de imagem. Revista Geminis, ano 7, n. 2, p. 136-153, 2016

ZHAO, Hengheng; HUANG, Ping; YAO, Junfeng. Texturing of Augmented Reality Character Based on Colored Drawing. IEEE Virtual Reality (VR), p. 355-356. USA, 2017. 